\title{
Microscopic morphology independence in linear absorption cross-section of $\mathrm{CsPbBr}_{3}$ nanocrystals
}

\author{
Fengying Zhang ${ }^{1,2,3}$, Yuchen $\mathrm{Liu}^{2}$, Shiqian $\mathrm{Wei}^{1}$, Junsheng $\mathrm{Chen}^{2}$, Ying Zhou ${ }^{1}$, Rongxing $\mathrm{He}^{3^{*}}$, \\ Tõnu Pullerits ${ }^{2^{*}}$ and Kaibo Zheng ${ }^{2,4^{*}}$
}

\begin{abstract}
Multiple morphologies of colloidal perovskite nanocrystals (NCs) diversify their optical and electronic properties. Among them, the linear absorption cross-section $(\sigma)$ is a primary parameter to determine their intrinsic photophysical features, and consequently, application potential. Herein, three morphologies of all-inorganic hybrid colloidal perovskite $\mathrm{CsPbBr}_{3} \mathrm{NCs}$, nanocubes (NBs), nanoplatelets (NLs), and nanowires (NWs), were targeted, and their linear $\sigma$ values were obtained through femtosecond transient absorption (TA) spectroscopy analysis. At high excitation energy well above the bandgap, the $\sigma$ per particle of all $\mathrm{CsPbBr}_{3} \mathrm{NCs}$ linearly increased with the particle volume $\left(V_{\mathrm{NC}}\right)$ regardless of the morphology with the value of $\sigma_{400}=9.45 \times 10^{4} \mathrm{~cm}^{-1} \times V_{\mathrm{NC}}$ $\left(\mathrm{cm}^{2}\right)$. Density functional theory (DFT) calculation confirmed the negligible influence of shapes on the optical selection rules. The Einstein spontaneous emission coefficients calculated from the $\sigma$ values define the intrinsic radiative recombination rate. However, reduced size dependence is observed when the excitation energy is close to the bandgap (i.e., at $460 \mathrm{~nm}$ ) with the value of $\sigma_{460}=2.82 \times 10^{8} \mathrm{~cm}^{0.65} \times\left(V_{\mathrm{NC}}\right)^{0.45}\left(\mathrm{~cm}^{2}\right)$. This should be ascribed to the discrete energy levels as well as lower density of states close to the band edge for perovskite NCs. These results provide in-depth insight into the optical characteristics for perovskite NCs.
\end{abstract}

Keywords: multiple morphologies, absorption cross-section, transient absorption spectroscopy, density functional theory, radiative recombination

\section{INTRODUCTION}

Lead-halide perovskites nanocrystals (NCs) have attracted immense attention in the past few years profiting from their prominent optoelectronic characteristics [1-4]. The bench-marked all-inorganic colloidal $\mathrm{CsPbX}_{3} \mathrm{NCs}(\mathrm{X}=\mathrm{I}$, $\mathrm{Br}, \mathrm{Cl})$ can achieve narrow photoluminescence (PL) emission and near-unity quantum yields with high stability. Besides, their optical absorption and emission can be conveniently adjusted via size and chemical composition engineering [5]. Recently, $\mathrm{CsPbX}_{3} \mathrm{NCs}$ with modified morphologies from conventional nanocubes (NBs) have been extensively investigated, involving onedimensional nanowires (1D NWs) and 2D nanoplatelets (2D NLs) [6-10]. The morphologic diversity of those nanostructures empowers the flexibility of device assembly. More importantly, the asymmetric quantum confinement can arise from those asymmetric structures fulfilling the special requirement of optoelectronic devices where anisotropic optical absorption or electric conduction is needed [11-13].

In order to elucidate the fundamental optical properties and provide guidance for the device application, lots of photophysical studies on the excited state dynamics, charge carrier transport, defect trapping, etc., have been discussed in recent literatures [14-17]. Most of these studies still utilized the primary optical parameters, especially the absorption cross-section $(\sigma)$ of bulk materials or conventional NBs. The $\sigma$ of $\mathrm{CsPbBr}_{3} \mathrm{NBs}$ and their size dependence have been extensively investigated in literature including our own previous studies [18-20]. While, the morphology dependence of light absorption in strongly quantum-confined systems (e.g., quantum dots, quantum wells) is so far not systematically investigated. Here the Coulomb interactions within confined excitons are responsible for additional renormalization effects

\footnotetext{
${ }^{1}$ State Key Laboratory of Oil and Gas Reservoir Geology and Exploitation and School of New Energy and Materials, Southwest Petroleum University, Chengdu 610500, China

2 Department of Chemical Physics and NanoLund Chemical Center, Lund University P.O. Box 124, 22100 Lund, Sweden

${ }^{3}$ College of Chemistry and Chemical Engineering, Southwest University, Chongqing 400715, China

${ }^{4}$ Department of Chemistry, Technical University of Denmark, DK-2800 Kongens Lyngby, Denmark

* Corresponding authors (emails: herx@swu.edu.cn (He R); tonu.pullerits@chemphys.lu.se (Pullerits T); kaibo.zheng@chemphys.lu.se (Zheng K))
} 
which can play important role [21,22]. In this scenario, the $\sigma$ values in other morphologies of NCs could deviate from the NBs due to their asymmetric local field factor as well as dielectric surrounding induced from the capping agent [21]. In addition, dominant exposure facets of NCs are morphology-dependent while the contribution of surface states can modulate the oscillator strength [23]. Therefore, it is indispensable to revisit the $\sigma$ of perovskite NCs with specific morphologies and provide accurate parameters for fundamental studies as well as device engineering.

Conventional approaches to evaluating the NCs $\sigma$, including ion concentration and gravimetric analysis, may cause uncertainty given the uneven NCs size distribution and disturbance from the surface ligands. Herein, a wellestablished method using femtosecond transient absorption (TA) spectroscopy was employed to characterize the $\sigma$ of three $\mathrm{CsPbBr}_{3} \mathrm{NCs}$ (NBs, NLs and NWs) per particle, which tracks the Poissonian distribution of photogenerated excitons at excited states [20,24-27]. We conclude that the $\sigma$ at high photon energy $(400 \mathrm{~nm})$ above the band edge strictly follows the bulk behavior and linearly scales up with the volume of the NCs regardless of the NC morphology with the relation of $\sigma_{400}=9.45 \times 10^{4} \mathrm{~cm}^{-1} \times$ $V_{\mathrm{NC}}\left(V_{\mathrm{NC}}\right.$ is the volume of a single NC in the unit of $\left.\mathrm{cm}^{3}\right)$. Very different size dependence was observed when the excitation energy is close to the absorption band edge $(460 \mathrm{~nm})$, which is ascribed to the discrete energy levels with less density of states (DOS) close to the band edge. To our surprise, $\sigma$ values at $460 \mathrm{~nm}$ exhibit the exponential dependence on the volume of NCs with the relation of $\sigma_{460}=2.82 \times 10^{8} \mathrm{~cm}^{0.65} \times\left(V_{\mathrm{NC}}\right)^{0.45}$ for three morphologies with different symmetry of the confinement. This is because in the direction of critical confined dimensions (i.e., the dimension in the direction with strongest quantum confinement regime) of NCs, the overlap between electron and hole wave functions does not change with size any more. Our findings unify the utilization of the $\sigma$ in colloidal perovskite NCs with various morphologies.

\section{RESULTS AND DISCUSSION}

Three types of colloidal $\mathrm{CsPbBr}_{3}$ NCs with different morphologies, including NBs, NLs and NWs, were synthesized by the typical hot-inject method $[5,28,29]$. The steady-state absorption and emission spectra of the three samples are presented in Fig. 1a with the absorption edge located at 503, 513 and $445 \mathrm{~nm}$, while PL emission peaks at 513, 518 and $473 \mathrm{~nm}$ for NBs, NLs, NWs, respectively. Transmission electron microscopy (TEM) images in Fig. $1 \mathrm{~b}$ confirmed the three distinct morphologies of those NCs, and the respective sizes are summarized in Fig. 1c. Herein, NWs displayed the most pronounced exciton peak in the absorption spectrum. In contrast, inconspicuous exciton peak can be observed in NBs, which is primarily correlated to the critical confined
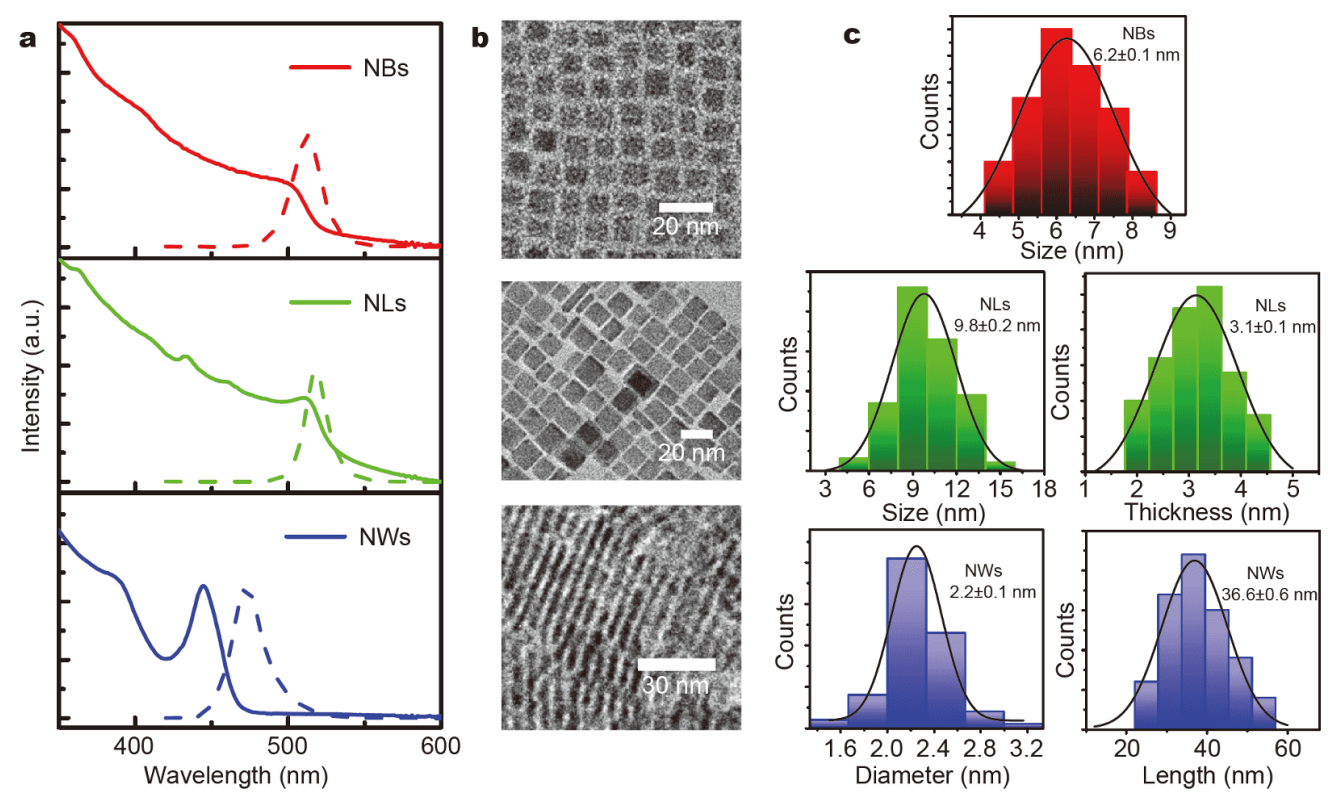

Figure 1 (a) Absorption (solid line), PL (dotted line) spectra and (b) TEM images of $\mathrm{CsPBrr}_{3}$ NCs with different morphologies. (c) The size distribution histogram for the key dimensions of $\mathrm{CsPbBr}_{3} \mathrm{NBs}$ and NLs and NWs, respectively. 
dimensions of the NCs. The excitons of NWs therefore are extremely quantum-confined in the radial direction with a diameter of $2.2 \mathrm{~nm}$, while the thickness of $3.1 \mathrm{~nm}$ is responsible for the confined excitons in NLs. As for 0D NBs, their excitons are evenly confined in three directions with an edge length of $\sim 6.2 \mathrm{~nm}$. Such critical dimension instead of the total volume determines the confinement regime as the volumes of NCs follow the trend of $V_{\mathrm{NLs}}>$ $V_{\mathrm{NBs}}>V_{\mathrm{NWs}}$.

To extract the linear $\sigma$ of quantum-confined NCs, we utilized the ultrafast TA spectroscopy, where the dynamics of the excited state population were probed (Figs S1 and S2). Fig. 2 presents the typical TA signals of the three different $\mathrm{CsPbBr}_{3}$ NCs. All samples featured distinct band-edge ground state bleach (GB) band (blue color). In addition, positive excited state absorption (ESA) appeared at the blue side of the GB of all three samples. According to the singular value decomposition (SVD) fitting, TA dynamics can be decomposed into three components as denoted in Fig. 2. The ultrafast component at femtosecond time-scale (400 to $500 \mathrm{fs}$ ) corresponds to the hot carrier cooling [30]. The component of tens to hundreds of picoseconds is ascribed to nonradiative recombination processes [20]. The third component on nanosecond timescale is the typical monomolecular radiative recombination of the photo- generated carriers $[20,31]$. The ratio of the amplitudes at the GB minimum between the second and third components almost complies with the photoluminescence quantum yield (PLQY) (Fig. S3), which justifies the assignment. In particular, the radiative recombination lifetimes of the three samples are $12 \mathrm{~ns}$ (NBs), $13 \mathrm{~ns}$ (NLs) and $3 \mathrm{~ns}$ (NWs), respectively, being consistent with the other reports in the literatures [32-34]. The fastest radiative recombination lifetime of $3 \mathrm{~ns}$ in $\mathrm{CsPbBr}_{3} \mathrm{NWs}$ should be attributed to the larger exciton binding energy evidenced by more pronounced band-edge exciton peak in Fig. 1a.

As the GB in TA measurement is induced by the state filling of the band edge exciton states, we can utilize the kinetics at GB to follow the evolution of exciton population in the NCs. Furthermore, the number of excitons per QD is discrete and the lifetimes corresponding to different numbers of excitons can be easily distinguished from TA kinetics. Disregarding the possible coherent effects caused by short laser pulses, the exciton population of NCs follows the Poissonian distribution [35-38]:

$P_{N}=\frac{\mathrm{e}^{-\langle N\rangle \cdot\langle N\rangle^{N}}}{N !}$,

where $P_{N}$ is the fraction of NCs with $N$ excitons, $N$ and $\langle N\rangle$ denote the number of excitons and the average exciton
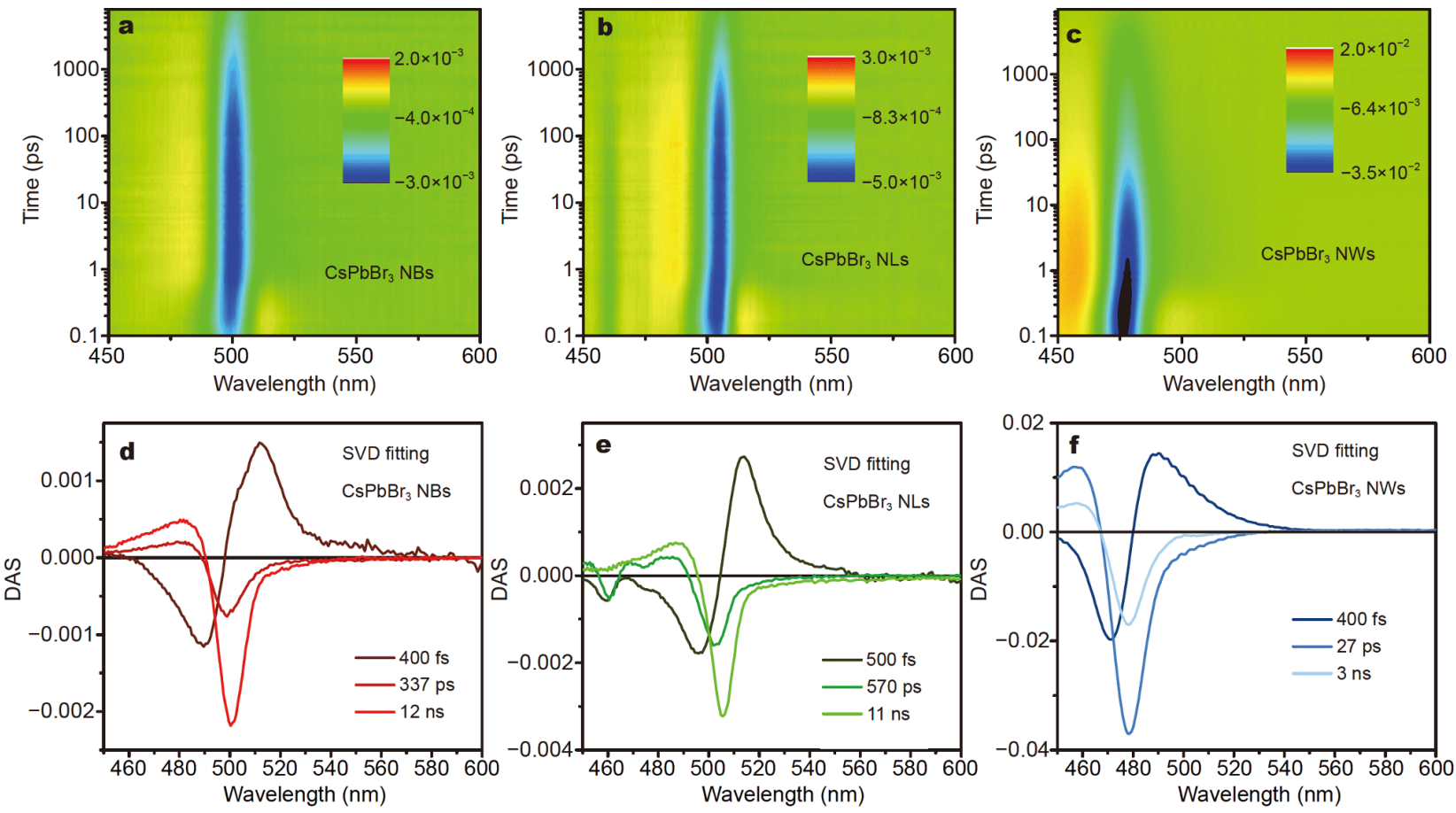

Figure 2 TA spectra of $\mathrm{CsPBrr}_{3}$ (a) NBs, (b) NLs and (c) NWs, and (d-f) the corresponding SVD fittings, at $300 \mathrm{~nm}$ excitation. 
number per NC, respectively. When the optical density of the sample is low, the excitation intensity does not vary in the sample volume and $\langle N\rangle$ can be expressed as

$\langle N\rangle=\sigma \cdot I$,

where $I$ is the excitation fluence (photons per pulse per excitation area) and $\sigma$ is the absorption cross-section at specific excitation wavelength. Combining Equations (1) and (2), the fraction of excited NCs can be calculated as follows [36]:

$P_{\text {exc }}=\sum_{N=1}^{\infty} P_{N}=1-P_{0}=1-\mathrm{e}^{-\langle N\rangle}=1-\mathrm{e}^{-\sigma \cdot I}$.

Multiple excitations in a QD decay through Auger process which is significantly faster than the radiative decay of the single excitation. This means that once the Auger process is completed, the signal amplitude is proportional to $P_{\text {exc }}$.

To eliminate the many-body effect, we fitted the linear region in the TA kinetics' logarithmic plot from the longtime delay and extrapolated such linear fitting to $t=0$ to get the $\Delta A_{0, \max }\left(\Delta A_{0, \max }\right.$ represents the highest singleexciton signal (all QDs excited) rescaled to $t=0$ ) that we need for the $\sigma$ calculation. The reason is when monomolecular recombination for the last remaining electron- hole pair dominates, it should reflect in TA kinetics as a single exponential decay and exhibit linearity in the logarithmic plot. At the early time scale, the TA kinetics deviates from this linearity due to the occurrence of highorder recombination processes such as Auger recombination in the QDs. As discussed, all excited $\mathrm{CsPbBr}_{3} \mathrm{NCs}$ contain a single exciton at long timescale [35]. In this scenario, $\Delta A(I)$ is merely proportional to $P_{\text {exc }}$ and decays mono-exponentially. We can rescale it to the corresponding signal at $t=0$, which is named as $\Delta A_{0}(I)$.

$\Delta A_{0}(I)=\frac{\Delta A(I)}{\mathrm{e}^{-t / \tau}}=\Delta A_{0, \max } \cdot\left(1-\mathrm{e}^{-\left(I / I_{0}\right) \cdot\langle N\rangle_{0}}\right)$.

Here the lowest pump intensity utilized in TA measurement was defined as $I_{0}$. We used such low intensity that the existence of multiple excitons in one NC can be neglected. Clearly, $\langle N\rangle_{0}$ can be obtained from the performed exponential fit of the $\Delta A_{0}(I) v s$. $\left(I / I_{0}\right)$ plots as described in Equation (4), and $\sigma$ can be calculated according to Equation (2). Fig. 3 presents the pump-intensity dependence of TA dynamics on the three types of NCs. As collected in Table S1, the calculated $\sigma$ values are $1.87 \times 10^{-14} \mathrm{~cm}^{2} \quad$ (NBs), $2.68 \times 10^{-14} \mathrm{~cm}^{2} \quad$ (NLs), $1.67 \times 10^{-14} \mathrm{~cm}^{2}$ (NWs) at the excitation wavelength of $400 \mathrm{~nm}$. When excitation wavelength is closer to the band
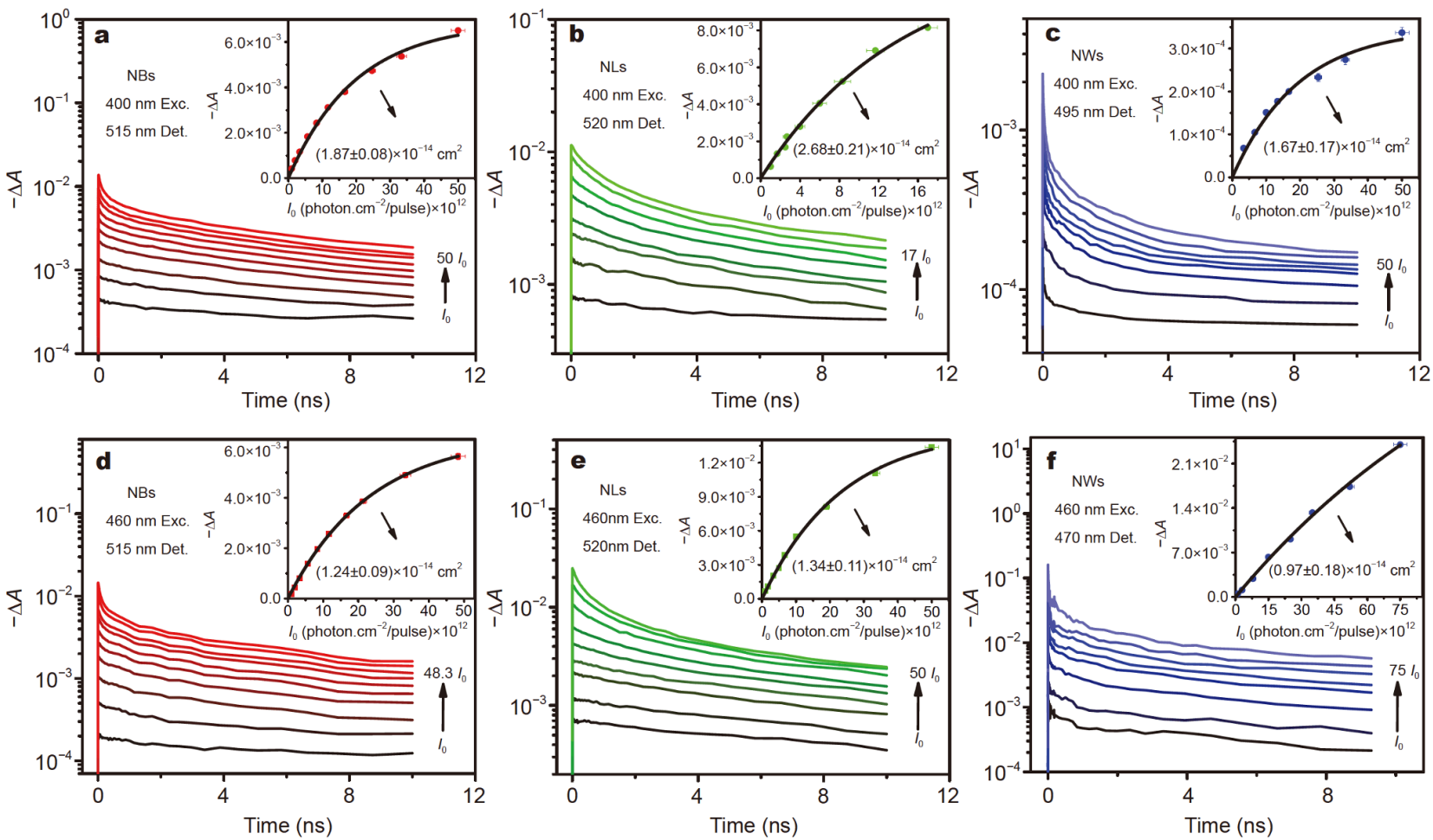

Figure 3 Pump-intensity dependence of TA dynamics for $\mathrm{CsPbBr}_{3} \mathrm{NBs}$, NLs and NWs, pumped at (a-c) $400 \mathrm{~nm}$ and (d-f) $460 \mathrm{~nm}$, respectively. 
edge (i.e., $460 \mathrm{~nm}$ ), the obtained $\sigma$ values were dropped to $1.24 \times 10^{-14}, 1.34 \times 10^{-14}$ and $0.97 \times 10^{-14} \mathrm{~cm}^{2}$, respectively.

The calculated $\sigma$ showed a perfect linear relationship $v s$. particle volume within the margin of error when $\mathrm{CsPbBr}_{3}$ NCs (NBs, NLs and NWs) were pumped at $400 \mathrm{~nm}$, as plotted in Fig. 4a. In addition, such a linear relationship complies again with the trend for NBs with different sizes. This indicates the absorption transition well above the optical band edge in $\mathrm{CsPbBr}_{3} \mathrm{NCs}$ is independent of the particle morphology and exhibits bulk-like behavior. The $\sigma$ value can be expressed as $\sigma_{400}=9.45 \times 10^{4} \mathrm{~cm}^{-1} \times V_{\mathrm{NC}}$ $\left(\mathrm{cm}^{2}\right)$ extracted from the slope in Fig. 4a. On the other hand, the $\sigma$ at wavelength closer to the optical band edge possesses reduced size dependence. To our surprise, those $\sigma$ values exhibited exponential dependence on the particle volume instead of critical dimensions within different morphologies, as presented in Fig. 4b.

In order to rationalize the morphology independence of the $\sigma$, we first calculated the DOS of $\mathrm{CsPbBr}_{3} \mathrm{NCs}$ with different morphologic lattice clusters corresponding to different preferential exposure facets as shown in Fig. 5 based on previous reports $[6,29,39]$. The conduction band minimum (CBM) and valence band maximum (VBM) in all three cases are mainly composed of $\mathrm{Pb} 6 \mathrm{p}$ and $\mathrm{Br} 4 \mathrm{p}$ orbitals, respectively. As the optical absorption in $\mathrm{CsPbr}_{3}$ perovskites is dominantly contributed by the optical transition electrons transfer from $\mathrm{Br} 4 \mathrm{p}$ to $\mathrm{Pb} 6 \mathrm{p}$ [40-42], such similar electronic structures indicate the conservation of angular momentum, energy, and linear momentum should be identical among all three samples during the optical transition. Therefore, we can conclude the morphology of $\mathrm{CsPbBr}{ }_{3} \mathrm{NCs}$ has a negligible effect on the optical selection rules. On the other hand, as well accepted in other quantum dot system, the DOS can be approximated as a continuum for absorption far above the band edge identical to the bulk counterparts (Fig. 4c) [43]. The above two factors elucidate the morphology independence of the $\sigma$ at high excitation photon energy.

At the regime close to the absorption band edge of the NCs, $\sigma$ is expected to deviate from bulk behavior as quantum confinement effect dominates. One well-accepted explanation is that the optical strength per NC of the firstexciton transition positively correlated to the particle volume will be canceled by the quantum confinement negatively correlated to the NC size $[44,45]$. This should be the main reason for the reduced size dependence of the $\sigma$ with $460 \mathrm{~nm}$ excitation as shown in Fig. 4b. The $\sigma$ value in this case can be expressed as $\sigma_{460}=2.82 \times 10^{8} \mathrm{~cm}^{0.65} \times$ $\left(V_{\mathrm{NC}}\right)^{0.45}\left(\mathrm{~cm}^{2}\right)$. Such size dependence on band edge $\sigma$ is weaker than that in most of semiconductor QDs (i.e., $\mathrm{CdSe}, \mathrm{PbSe}, \mathrm{PbS}$ ) [46-48]. What surprises us is that the $\sigma$ is in exponential dependence of particle volume for all three morphologies with different symmetry of the confinement. The main reason should be that the oscillation strength in strong quantum confinement regime is dimension-irrelevant on the overlap between wave functions of electron and hole [49]. In this regard, we can expect that the overlap of wave functions in the perovskite NCs is fixed as long as one critical dimension reaches strong quantum confinement regime as indicated in Fig. $4 \mathrm{c}$ and cannot deviate the oscillation strength.

The $\sigma$ at other wavelength of $\mathrm{CsPbBr}_{3}$ NCs can be extrapolated from steady-state absorption spectra (Fig. S4). We can now calculate the Einstein spontaneous emission coefficient $\left(A_{i}\right)$ according to the integrated $\sigma$ over the firstexciton band using Gaussian function as follows [50,51]: $A_{i}=\frac{8 \pi}{\lambda^{2}} \int_{v_{1}}^{v_{2}} \sigma(v) \mathrm{d} v$
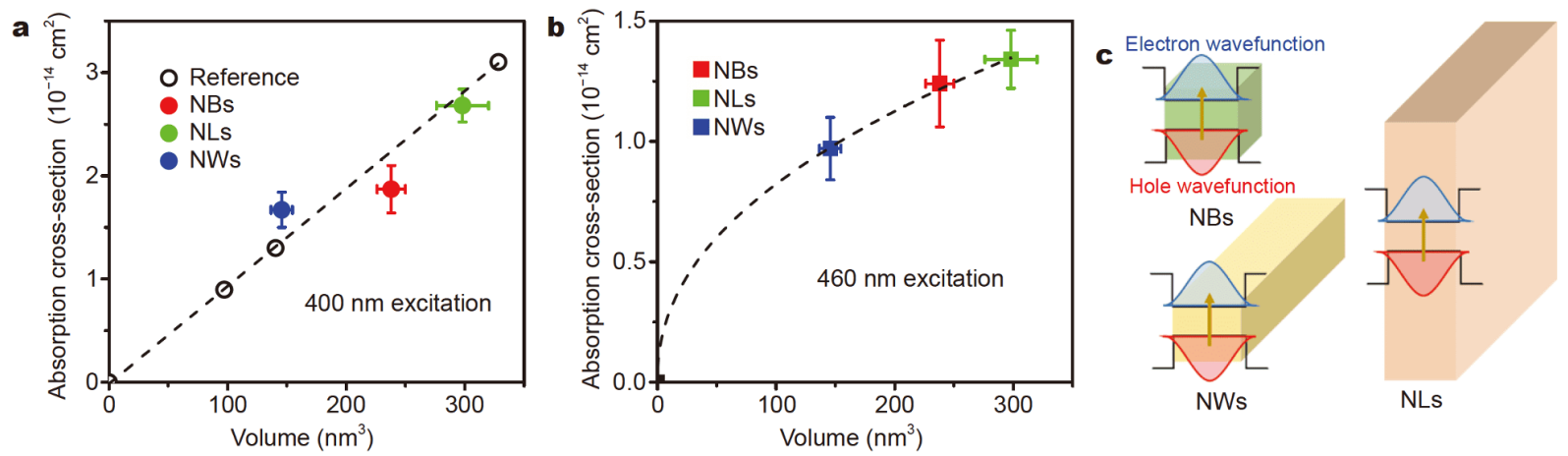

Figure 4 The absorption cross-section of $\mathrm{CsPbBr}_{3} \mathrm{NBs}$, NLs and NWs as a function of NCs volume with (a) $400 \mathrm{~nm}$ excitation and (b) $460 \mathrm{~nm}$ excitation, respectively. The hollow black circle represents the trend in $\mathrm{CsPbBr}_{3} \mathrm{NBs}$ with different sizes. The error bar for the volume is calculated from NC size distribution. The error bar for $\sigma$ is propagated from the error of excitation fluence and $-\Delta A / A$. (c) The schematic of overlap between electron and hole wave functions in NCs with different morphologies. 

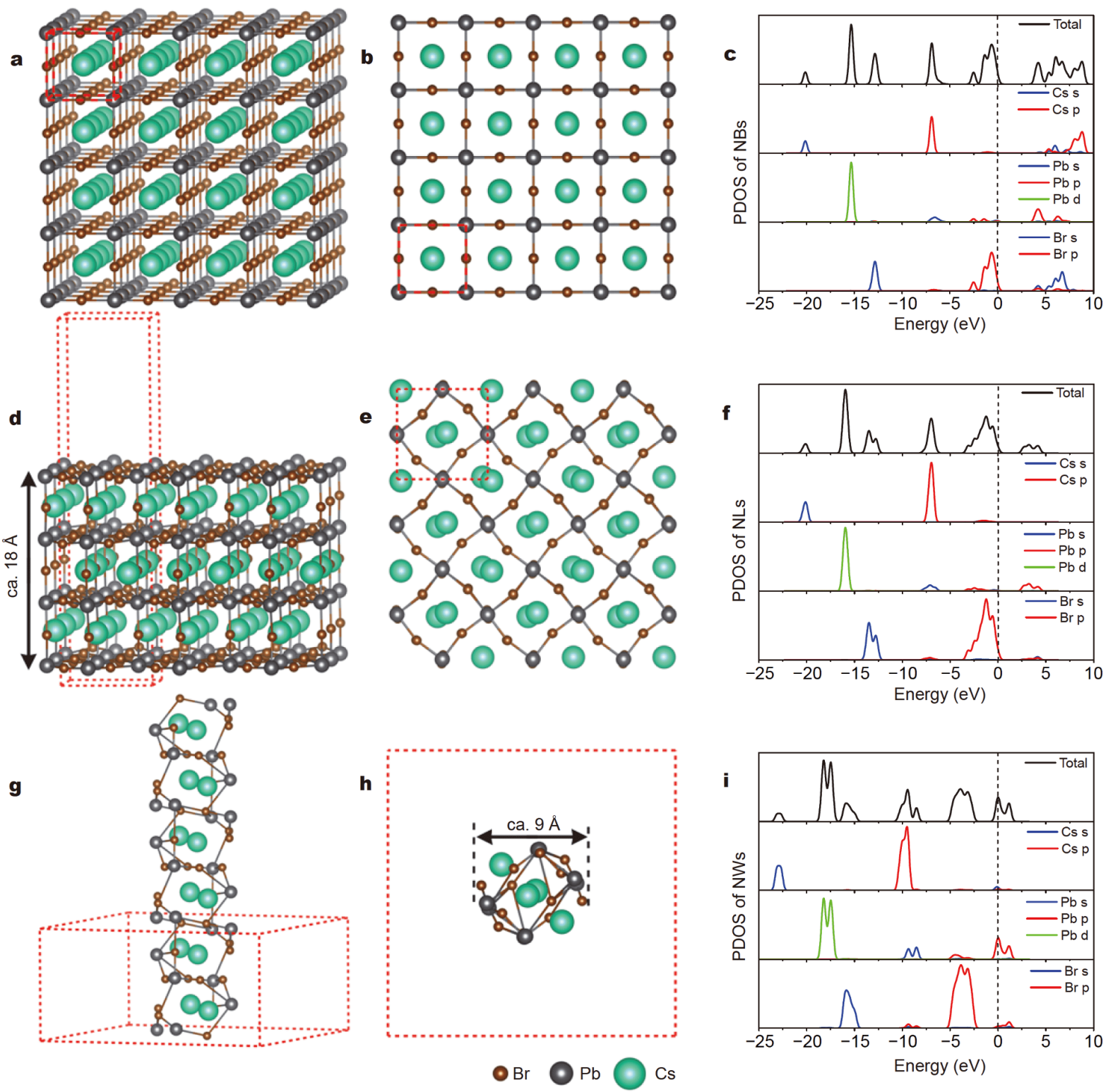

Figure 5 The side and top views of calculation model, as well as calculated DOS for (a-c) CsPbBr ${ }_{3} \mathrm{NBs}$, (d-f) NLs and (g-i) NWs, respectively. The unit cells of $\mathrm{Cs} \mathrm{PbBr}_{3}$ NCs are highlighted with red dashed lines.

where $\lambda$ represents the wavelength in centimeter for the first-exciton transition peak, $v_{1}$ and $v_{2}$ denote the start and end frequencies in Hertz at first-exciton absorption band. Furthermore, the first-exciton radiative lifetime $\tau$ for $\mathrm{CsPbBr}_{3}$ NCs with different morphologies was obtained from the inverse of $A_{i}$ [51].

$\tau=\frac{1}{A_{i}}$.

This calculated $\tau$ value can predict the intrinsic radiative lifetime in a given system. Here, both the calculated radiative lifetimes and the experimental results are listed in Table S2 and plotted in Fig. 6. The experimental values from TA measurement are significantly longer than the calculated lifetimes via using $A_{i}$, though, the similar trend among three samples, namely NBs $>$ NLs $>$ NWs still holds. We explain the discrepancy by the fact that the absorption of a semiconductor nanocrystal consists of many energetically overlapping transitions which all contribute to the $A_{i}$ calculation. At the same time, emission is induced by a single transition. This conclusion is well supported by the atomistic calculations of the 


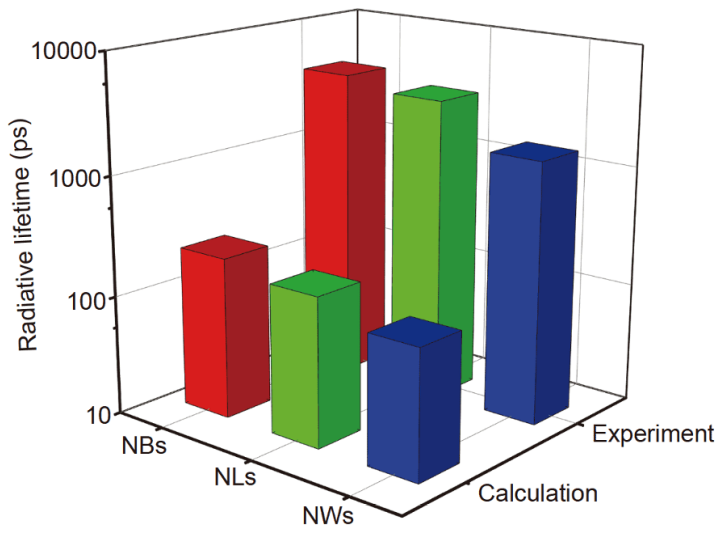

Figure 6 The calculated lower limit of radiative lifetimes and the experimental results for $\mathrm{CsPBr}_{3} \mathrm{NBs}$, NLs and NWs measured from TA studies, respectively.

semiconductor nanocrystals [52]. Furthermore, many processes prior to the 'true' radiative recombination including the diffusion of the charge carriers, trapping and detrapping from the shallow trap states, or the darkbright state splitting at the band edge of the nanoparticles can all prolong the lifetime measured in TA [53].

\section{CONCLUSIONS}

We have successfully synthesized $\mathrm{CsPbBr}{ }_{3} \mathrm{NCs}$ with three morphologies by the hot injection method, including NBs, NPs, and NWs with different aspect ratios. The linear $\sigma$ values per NC are calculated from the excited state depopulation kinetics based on TA studies. Results showed that the value of $\sigma$ at high photon energy above the band edge strictly follows the bulk-like behavior and linearly scales up with the volume of NCs regardless of the morphologies. This is due to the bulk-like continuum band structure at high energy and morphologic independent optical band transition visualized from DFT calculation. When the photon energy is close to the optical band edge, the $\sigma$ exhibits reduced size dependence due to the enhanced quantum confinement. However, such confinement effect is also morphologic (or aspect ratio) independent, whereas it is more dominated by the reduced dependence of NC volume. Our observation indicates the utilization of basic absorption parameters from conventional NBs in other morphologies of colloidal $\mathrm{CsPbBr}_{3}$ nanostructures is practicable without worrying about the surface or dimensional effects.

Received 21 September 2020; accepted 2 November 2020; published online 13 January 2021

1 Li X, Bi D, Yi C, et al. A vacuum flash-assisted solution process for high-efficiency large-area perovskite solar cells. Science, 2016, 353: $58-62$

2 Stranks SD, Snaith HJ. Metal-halide perovskites for photovoltaic and light-emitting devices. Nat Nanotech, 2015, 10: 391-402

3 Snaith HJ. Present status and future prospects of perovskite photovoltaics. Nat Mater, 2018, 17: 372-376

4 Chen J, Du W, Shi J, et al. Perovskite quantum dot lasers. InfoMat, 2020, 2: 170-183

5 Protesescu L, Yakunin S, Bodnarchuk MI, et al. Nanocrystals of cesium lead halide perovskites $\left(\mathrm{CsPbX}_{3}, \mathrm{X}=\mathrm{Cl}, \mathrm{Br}\right.$, and I): Novel optoelectronic materials showing bright emission with wide color gamut. Nano Lett, 2015, 15: 3692-3696

6 Seth S, Samanta A. A facile methodology for engineering the morphology of $\mathrm{CsPb}_{3}$ perovskite nanocrystals under ambient condition. Sci Rep, 2016, 6: 37693

7 Liang Z, Zhao S, Xu Z, et al. Shape-controlled synthesis of allinorganic $\mathrm{CsPbBr}$ perovskite nanocrystals with bright blue emission. ACS Appl Mater Interfaces, 2016, 8: 28824-28830

8 Sun S, Yuan D, Xu Y, et al. Ligand-mediated synthesis of shapecontrolled cesium lead halide perovskite nanocrystals via reprecipitation process at room temperature. ACS Nano, 2016, 10: 3648-3657

9 Di Stasio F, Imran M, Akkerman QA, et al. Reversible concentration-dependent photoluminescence quenching and change of emission color in $\mathrm{CsPBr}_{3}$ nanowires and nanoplatelets. J Phys Chem Lett, 2017, 8: 2725-2729

10 Jeon S, Jung MC, Ahn J, et al. Post-synthetic oriented attachment of $\mathrm{CsPbBr}_{3}$ perovskite nanocrystal building blocks: From first principle calculation to experimental demonstration of size and dimensionality (0D/1D/2D). Nanoscale Horiz, 2020, 5: 960-970

11 Li Q, Lian T. Ultrafast charge separation in two-dimensional $\mathrm{CsPbBr}_{3}$ perovskite nanoplatelets. J Phys Chem Lett, 2019, 10: 566573

12 Yang D, Zou Y, Li P, et al. Large-scale synthesis of ultrathin cesium lead bromide perovskite nanoplates with precisely tunable dimensions and their application in blue light-emitting diodes. Nano Energy, 2018, 47: 235-242

13 Shang Q, Li C, Zhang S, et al. Enhanced optical absorption and slowed light of reduced-dimensional $\mathrm{CsPbBr}_{3}$ nanowire crystal by exciton-polariton. Nano Lett, 2020, 20: 1023-1032

14 Zhang $\mathrm{X}, \mathrm{Wu} \mathrm{X}$, Liu X, et al. Heterostructural $\mathrm{CsPbX}_{3}-\mathrm{PbS}(\mathrm{X}=\mathrm{Cl}$, $\mathrm{Br}, \mathrm{I})$ quantum dots with tunable Vis-NIR dual emission. J Am Chem Soc, 2020, 142: 4464-4471

15 Chen J, Zhang Q, Shi J, et al. Room temperature continuous-wave excited biexciton emission in perovskite nanoplatelets via plasmonic nonlinear fano resonance. Commun Phys, 2019, 2: 80

16 Chen W, Zhang S, Zhou M, et al. Two-photon absorption-based upconverted circularly polarized luminescence generated in chiral perovskite nanocrystals. J Phys Chem Lett, 2019, 10: 3290-3295

17 Yang B, Han K. Charge-carrier dynamics of lead-free halide perovskite nanocrystals. Acc Chem Res, 2019, 52: 3188-3198

18 Maes J, Balcaen L, Drijvers E, et al. Light absorption coefficient of $\mathrm{CsPBBr}_{3}$ perovskite nanocrystals. J Phys Chem Lett, 2018, 9: 30933097

19 Puthenpurayil J, Cheng OHC, Qiao T, et al. On the determination of absorption cross section of colloidal lead halide perovskite quantum dots. J Chem Phys, 2019, 151: 154706

20 Chen J, Žídek K, Chábera P, et al. Size- and wavelength-dependent two-photon absorption cross-section of $\mathrm{CsPbBr}_{3}$ perovskite quantum dots. J Phys Chem Lett, 2017, 8: 2316-2321 
21 Hens Z, Moreels I. Light absorption by colloidal semiconductor quantum dots. J Mater Chem, 2012, 22: 10406-10415

22 Chu S. Cold atoms and quantum control. Nature, 2002, 416: 206210

23 Huang MH. Facet-dependent optical properties of semiconductor nanocrystals. Small, 2019, 15: 1804726

24 Htoon H, Hollingsworth JA, Dickerson R, et al. Effect of zero- to one-dimensional transformation on multiparticle auger recombination in semiconductor quantum rods. Phys Rev Lett, 2003, 91: 227401

25 Ivanov SA, Achermann M. Spectral and dynamic properties of excitons and biexcitons in type-II semiconductor nanocrystals. ACS Nano, 2010, 4: 5994-6000

26 Wang Y, Li X, Song J, et al. All-inorganic colloidal perovskite quantum dots: A new class of lasing materials with favorable characteristics. Adv Mater, 2015, 27: 7101-7108

27 Zhang D, Yu Y, Bekenstein Y, et al. Ultrathin colloidal cesium lead halide perovskite nanowires. J Am Chem Soc, 2016, 138: 1315513158

28 Pan $\mathrm{A}, \mathrm{He} \mathrm{B}$, Fan $\mathrm{X}$, et al. Insight into the ligand-mediated synthesis of colloidal $\mathrm{CsPbBr}_{3}$ perovskite nanocrystals: The role of organic acid, base, and cesium precursors. ACS Nano, 2016, 10: 7943-7954

29 Imran M, Di Stasio F, Dang Z, et al. Colloidal synthesis of strongly fluorescent $\mathrm{CsPbBr}_{3}$ nanowires with width tunable down to the quantum confinement regime. Chem Mater, 2016, 28: 6450-6454

30 Chen J, Messing ME, Zheng K, et al. Cation-dependent hot carrier cooling in halide perovskite nanocrystals. J Am Chem Soc, 2019, 141: $3532-3540$

31 Zhang F, Chen J, Zhou Y, et al. Effect of synthesis methods on photoluminescent properties for $\mathrm{CsPbBr}_{3}$ nanocrystals: Hot injection method and conversion method. J Lumin, 2020, 220: 117023

32 Li Y, Ding T, Luo X, et al. Biexciton auger recombination in monodispersed, quantum-confined $\mathrm{CsPBBr}_{3}$ perovskite nanocrystals obeys universal volume-scaling. Nano Res, 2019, 12: 619-623

33 Wang $\mathrm{L}$, Fu K, Sun $\mathrm{R}$, et al. Ultra-stable $\mathrm{CsPbBr}_{3}$ perovskite nanosheets for X-ray imaging screen. Nano-Micro Lett, 2019, 11: 52

34 Zhou $\mathrm{H}$, Song Z, Grice CR, et al. Self-powered $\mathrm{CsPbBr}_{3}$ nanowire photodetector with a vertical structure. Nano Energy, 2018, 53: 880-886

35 Makarov NS, Guo S, Isaienko O, et al. Spectral and dynamical properties of single excitons, biexcitons, and trions in cesium-leadhalide perovskite quantum dots. Nano Lett, 2016, 16: 2349-2362

36 Lenngren N, Garting T, Zheng K, et al. Multiexciton absorption cross sections of CdSe quantum dots determined by ultrafast spectroscopy. J Phys Chem Lett, 2013, 4: 3330-3336

37 Klimov VI, Mikhailovsky AA, McBranch DW, et al. Quantization of multiparticle auger rates in semiconductor quantum dots. Science, 2000, 287: 1011-1013

38 Ji M, Park S, Connor ST, et al. Efficient multiple exciton generation observed in colloidal PbSe quantum dots with temporally and spectrally resolved intraband excitation. Nano Lett, 2009, 9: 12171222

39 Shamsi J, Dang Z, Bianchini $\mathrm{P}$, et al. Colloidal synthesis of quantum confined single crystal $\mathrm{CsPbr}_{3}$ nanosheets with lateral size control up to the micrometer range. J Am Chem Soc, 2016, 138: 7240-7243

40 Yang D, Li X, Zhou W, et al. $\mathrm{CsPbBr}_{3}$ quantum dots 2.0: Benzenesulfonic acid equivalent ligand awakens complete purification.
Adv Mater, 2019, 1900767

41 Wan W, Zhou YY, Yan L, et al. In situ compositing $\mathrm{CsPbBr}_{3}$ with exfoliated layered-perovskite $\mathrm{CsCa}_{2} \mathrm{Ta}_{3} \mathrm{O}_{10}$ : Interfacial interaction and enhanced stability. ACS Appl Mater Interfaces, 2019, 11: 47227-47236

42 Goesten MG, Hoffmann R. Mirrors of bonding in metal halide perovskites. J Am Chem Soc, 2018, 140: 12996-13010

43 Leatherdale CA, Woo WK, Mikulec FV, et al. On the absorption cross section of CdSe nanocrystal quantum dots. J Phys Chem B, 2002, 106: 7619-7622

44 Brus LE. Electron-electron and electron-hole interactions in small semiconductor crystallites: The size dependence of the lowest excited electronic state. J Chem Phys, 1984, 80: 4403-4409

45 Hanamura E. Very large optical nonlinearity of semiconductor microcrystallites. Phys Rev B, 1988, 37: 1273-1279

46 Yu WW, Qu L, Guo W, et al. Experimental determination of the extinction coefficient of CdTe, CdSe, and CdS nanocrystals. Chem Mater, 2003, 15: 2854-2860

47 Cademartiri L, Montanari E, Calestani G, et al. Size-dependent extinction coefficients of PbS quantum dots. J Am Chem Soc, 2006, 128: $10337-10346$

48 Moreels I, Lambert K, De Muynck D, et al. Composition and sizedependent extinction coefficient of colloidal PbSe quantum dots. Chem Mater, 2007, 19: 6101-6106

49 Leistikow MD, Johansen J, Kettelarij AJ, et al. Size-dependent oscillator strength and quantum efficiency of CdSe quantum dots controlled via the local density of states. Phys Rev B, 2009, 79: 045301

50 Hilborn RC. Einstein coefficients, cross sections, $f$ values, dipole moments, and all that. Am J Phys, 1982, 50: 982-986

51 Yu P, Beard MC, Ellingson RJ, et al. Absorption cross-section and related optical properties of colloidal InAs quantum dots. J Phys Chem B, 2005, 109: 7084-7087

52 Prezhdo OV. Multiple excitons and the electron-phonon bottleneck in semiconductor quantum dots: An $a b$ initio perspective. Chem Phys Lett, 2008, 460: 1-9

53 Fisher BR, Eisler HJ, Stott NE, et al. Emission intensity dependence and single-exponential behavior in single colloidal quantum dot fluorescence lifetimes. J Phys Chem B, 2004, 108: 143-148

Acknowledgements This work was supported by the National Natural Science Foundation of China (NSFC, U1862111), China Scholarship Council (201706990062), Independent Research Fund Denmark-Nature Sciences (DFF-7014-00302), Independent Research Fund DenmarkSapere Aude starting grant (7026-00037A), Swedish Research Council VR starting grant (2017-05337), grants VR2018-06011, and VR201805090, the Research Fund for international Young Scientists from NSFC, China (21950410515) and Swedish Energy Agency.

Author contributions Zhang F designed and engineered the samples; Liu Y, Wei S and Chen J assisted to perform the experiments; Zhou Y, He R and Pullerits T helped to modify the manuscript; Zheng $\mathrm{K}$ led the project.

Conflict of interest The authors declare that they have no conflict of interest.

Supplementary information Experimental details and supporting data are available in the online version of the paper. 


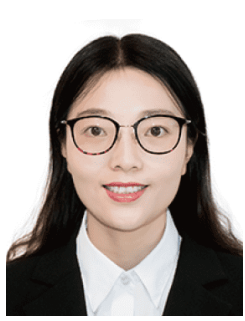

Fengying Zhang received her $\mathrm{PhD}$ degree from Southwest University in 2019. During her PhD period, she studied at Lund University as a visiting $\mathrm{PhD}$, dedicated to the study of material spectroscopy. Then, she joined the School of New Energy and Materials, Southwest Petroleum University as a lecturer, and has engaged in material-related scientific research.

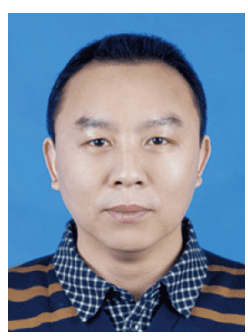

Rongxing $\mathrm{He}$ is a professor at Southwest University, China. He received his $\mathrm{PhD}$ from Sichuan University in 2006. Then, he joined the School of Chemistry and Chemical Engineering, Southwest University. His main research interests include molecular spectroscopy and photochemistry, electrochemical catalysis and reaction mechanism, and photoelectric functional materials.

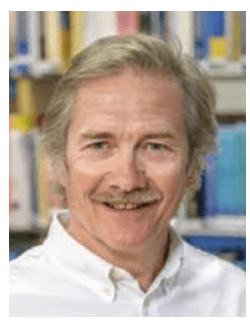

Tõnu Pullerits obtained his $\mathrm{PhD}$ degree in 1991 from the Institute of Physics at Tartu University, Estonia. He is currently full professor and head of the Chemical Physics Division at Lund University. $\mathrm{He}$ has been a member of the Royal Swedish Academy of Sciences since 2016. His research interests include energy transport in molecular systems, ultrafast charge carrier dynamics and photophysics in photovoltaic materials, and coherence multidimensional spectroscopy.

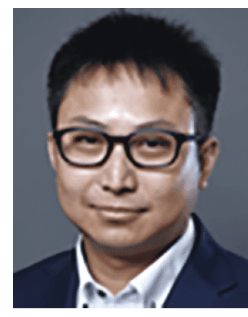

Kaibo Zheng obtained his PhD degree in 2010 from the Department of Materials Science at Fudan University. Then, he joined the Chemical Physics Division at Lund University, Sweden, as a postdoctoral fellow. He is currently a senior researcher in chemical physics, Lund University, and dual affiliated as a senior researcher at the Department of Chemistry, Technical University of Denmark. His research interests include the structure and photophysics of semiconductor and perovskite quantum dots as light harvesting materials.

\section{$\mathrm{CsPbBr}_{3}$ 纳米晶微观形貌非依赖的线性吸收截面}

张凤英 ${ }^{1,2,3}$, 刘禹辰 ${ }^{2}$, 卫诗倩 ${ }^{1}$, 陈俊生 $^{2}$, 周莹 ${ }^{1}$, 何荣幸 ${ }^{3}$,

Tõnu Pullerits ${ }^{2^{*}}$, 郑凯波 ${ }^{2,4}$

摘要 胶体钙钛矿纳米晶的形貌变化赋予其不同的光学和电子特 性, 其中, 线性吸收截面是决定材料本征光物理特性及其应用潜力 的主要参数. 本文研究了三种不同形貌的全无机钙钛矿 $\mathrm{Cs} \mathrm{PbBr}_{3}$ 纳 米晶(纳米立方体、纳米片和纳米线), 通过飞秒瞬态吸收光谱分析 了它们的线性吸收截面值 $\sigma$. 在高于带隙的能量激发下, 单个 $\mathrm{CsPbBr}_{3}$ 纳米晶的吸收截面值与形貌无关, 且随着粒子的体积 $\left(V_{\mathrm{NC}}\right)$ 呈 $\sigma_{400}=9.45 \times 10^{4} \mathrm{~cm}^{-1} \times V_{\mathrm{NC}}\left(\mathrm{cm}^{2}\right)$ 趋势变化. 密度泛函理论证实 了形貌对光学选择规则基本没有影响, 同时, 根据吸收截面值也计 算得到了定义本征辐射复合率的爱因斯坦自发辐射系数. 然而, 当 激发能接近带隙 $(460 \mathrm{~nm})$ 时, 吸收截面值对纳米晶尺寸的依赖关系 变弱, 取值满足 $\sigma_{460}=2.82 \times 10^{8} \mathrm{~cm}^{0.65} \times\left(V_{\mathrm{NC}}\right)^{0.45}\left(\mathrm{~cm}^{2}\right)$, 这主要归因 于钲钛矿纳米晶的离散能级及靠近带边的较低的态密度. 本文的 研究结果为洞悉钻铁矿纳米晶的光学特性提供了深入的见解. 